\title{
Design and Performance of Viral Clearance Studies with Tissue-Derived Products
}

\author{
By Stephen Stoltzfus and Katherine F. Bergmann
}

\section{Introduction}

$\mathbf{T}$

issue-derived products are a class of biological materials harvested directly from animal or human tissue, in contrast to recombinant DNA materials grown in cell culture bioreactors. Tissue-derived products are often used for structural purposes and are typically regulated as medical devices. However, when used to treat human patients, tissue-derived products are subject to many of the same concerns as recombinant DNA biotherapeutics, with viral safety being one of them. To address this, the tissue source material must undergo a risk analysis and testing regimen for the presence of viral contaminants. In addition, viral clearance studies must be performed to evaluate whether the purification process is robust enough to remove and/or inactivate viruses that may be present in the starting material.

The goals of viral clearance studies are the same for tissuederived products and biotherapeutics, but the design and performance of these studies can be quite different because of the diverse nature of the materials. In this article, we will present an overview of viral clearance studies for tissue-derived products based on our experience in performing a large number of such studies. Rather than discussing the issues related to viral clearance in general, our focus will be on the unique challenges that tissue-derived products pose.

\section{Regulatory Guidance}

The primary regulatory guidance for viral safety evaluation of biotechnological products comes from the International Conference on Harmonisation (ICH Q5A). In addition, both the United States Food and Drug Administration (US FDA) and the European Medicines Agency (EMA) have issued guidance on viral clearance. Regulatory guidance related to human tissuederived products is shown in Table 1. Unfortunately, there is nothing specific to animal tissue-derived products or that focuses on viral clearance for these products from any species. The principles of ICH Q5A are generally followed for viral clearance studies with tissue-derived products (e.g., heart valves, musculoskeletal components, organs, etc.). However, because of the many differences between tissue-derived products and recombinant DNA therapeutics in suspension, the expectations for viral clearance can be very different. For example, it is challenging to determine the risk of virus contamination that could be present in a herd as opposed to the well-established risk profile of cell culture bioreactors. Plus there may be only a limited number of suitable processing steps that can lead to virus removal and/or inactivation. Therefore, the expected clearance target is lower than what is expected for biotherapeutics.

\section{TABLE 1. Regulatory guidance on \\ viral clearance in biological products.}

International Conference on Harmonisation. ICH Q5A (R1) Quality of biotechnological products: viral safety evaluation of biotechnology products derived from cell lines of human or animal origin. (PMP/ ICH/295/9. Oct 1997. https://www.ema.europa.eu/en/documents/ scientific-guideline/ich-q-5-r1-viral-safety-evaluation-biotechnology-products-derived-cell-lines-human-animal-origin_en.pdf

Center for Biologics Evaluation and Research, US Food and Drug Administration (FDA CBER). Points to consider in the characterization of cell lines used to produce biologicals. 12 July 1993. https://www.fda.gov/ media/76255/download

Center for Biologics Evaluation and Research, US Food and Drug Administration (FDA CBER). Points to consider in the manufacture and testing of monoclonal antibody products for human use. 28 Feb 1997. https://www.fda.gov/media/76798/download

Committee for Proprietary Medicinal Products (CPMP) and the Biotechnology Working Party (BWP). Note for guidance on virus validation studies: The design, contribution and interpretation of studies validating the inactivation and removal of viruses. CPMP/BWP/268/95. 3-4 July 1995. https://www.ema. europa.eu/en/documents/scientific-guideline/note-guidance-virus-validation-studies-design-contribution-interpretation-studies-validating_en.pdf Committee for Human Medicinal Products (CHMP) and the Biotechnology Working Party (BWP). Guideline on virus safety evaluation of biotechnological investigational medicinal products. EMEA/CHMP/BWP/398498/2005. 24 July 2008. https://www.ema.europa.eu/en/documents/scientific-guideline/ guideline-virus-safety-evaluation-biotechnological-investigational-medicinal-products_en.pdf

Center for Biologics Evaluation and Research, US Food and Drug Administration (FDA CBER). Guidance for industry: Current good tissue practice (CGTP) and additional requirements for manufacturers of human cells, tissues, and cellular and tissue-based products (HCT/Ps). December 2011. https://www.fda.gov/media/82724/download

Center for Devices and Radiological Health, US Food and Drug Administration (FDA CDRH). Medical devices containing materials derived from animal sources (except for in vitro diagnostic devices): Guidance for industry and Food and Drug Administration staff. 15 March 2019. https://www.fda. gov/media/87251/download

International Organization for Standardization (ISO). ISO 22442-1:2015. Medical devices utilizing animal tissues and their derivatives - Part 1: Application of risk management. November 2015. https://www.iso.org/ standard/68553.html

International Organization for Standardization (ISO). ISO 22442-2:2015. Medical devices utilizing animal tissues and their derivatives - Part 2: Controls on sourcing, collection and handling. November 2015. https:// www.iso.org/standard/68554.html

International Organization for Standardization (ISO). IS0 22442-3:2007. Medical devices utilizing animal tissues and their derivatives - Part 3: Validation of the elimination and/or inactivation of viruses and transmissible spongiform encephalopathy (TSE) agents. December 2007. https://www. iso.org/standard/39351.html 


\section{The Starting Material}

Tissue-derived products are usually solid in consistency. Collagen-based products that are solubilized or rendered into a slurry during processing are one exception. Since biotherapeutics are produced in liquid form, purification and viral clearance studies are performed in solution. Undertaking such studies with non-liquids is difficult, especially during virus spiking, recovery, and determining accurate viral titers.

The source of the material can present challenges as well. Many animal-derived products are sourced from food-grade animals in the US, and there are no issues with the use of this material. If tissue is sourced from other animals, a USDA permit may be required in order to ship the material to the testing site. If the tissue is sourced from outside the US, a USDA permit will be required, and this may be impossible to obtain depending on the country of origin.

\section{Tissue Processing Steps}

Most tissue-derived products are not highly purified and may be heterogeneous in nature. They are usually subjected to a series of treatments and extractions to remove unwanted components such as cells or lipids. The treatments shown in Table 2 can potentially achieve some degree of viral inactivation, but it all depends on the virus and which processing steps are used. In addition, many processing steps involve tissue washing, which are not usually considered very effective or robust in inactivating or removing virus.

Manufacturers focus heavily on removing unnecessary byproducts while maintaining tissue functionality, and don't always acknowledge the need for effective viral clearance steps, which can create several issues:

- There may be multiple processing steps utilizing the same component, such as a detergent. Because the regulatory guidance indicates that clearance steps must be orthogonal, two steps with a common clearance mechanism cannot both be claimed.

- The process may not include any steps having the potential to remove or inactivate virus. As a result, it may not be possible to measure enough virus reduction to satisfy regulatory requirements.

\begin{tabular}{|l|l|}
\hline \multicolumn{2}{|c|}{$\begin{array}{c}\text { TABLE 2. Treatments for viral clearance commonly } \\
\text { used in the processing of tissue-derived products. }\end{array}$} \\
\hline \multicolumn{1}{|c|}{ Processing Step } & \multicolumn{1}{c|}{$\begin{array}{c}\text { Typical Viral Clearance } \\
\left.\text { Values (log }{ }_{10}\right)^{*}\end{array}$} \\
\hline Low pH treatment & $>4(\mathrm{pH} \sim 1)$ \\
\hline High pH treatment & $>4(\mathrm{pH}>12)$ \\
\hline Solvent extraction & $>4($ enveloped viruses only) \\
\hline Detergent treatment & $>4$ (enveloped viruses only) \\
\hline Treatment with oxidizing agent & $>4$ \\
\hline Heat treatment & Process and virus dependent \\
\hline Gamma irradiation & Process and virus dependent \\
\hline Electron beam irradiation & Process and virus dependent \\
\hline \multicolumn{2}{|c|}{ *Typical values derived from our numerous studies. Data not shown. } \\
\hline
\end{tabular}

- One processing step may contain residual inactivant agents from the previous step. In order to test the step in isolation, the tissue must be washed to remove the residual. Although the washing step may not be part of the manufacturing process, it is necessary in order to separate the two processing steps for evaluation of viral clearance.

The treatments shown in Table $\mathbf{2}$ are detailed here:

- Low pH treatment. Depending on the pH level used for inactivation, this step may be effective for all viruses. However, we have seen a number of occasions when the inactivation was lower than expected when compared to solution studies. For example, treatment at $\mathrm{pH} 2.0-2.5$ is highly effective for enveloped viruses in solution. However, this range may not be completely effective with tissuebased products, as the tissue appears to protect the virus from inactivation. It may be necessary to use a lower $\mathrm{pH}$ to achieve effective clearance.

- High pH treatment. Again, the effectiveness depends upon the $\mathrm{pH}$. The $\mathrm{pH}$ must be higher than 11 to inactivate parvoviruses. At this $\mathrm{pH}$, most other viruses will be inactivated effectively.

- Solvent or detergent treatment. Both of these are usually effective in inactivating enveloped viruses, but they are typically ineffective for non-enveloped viruses.

- Heat treatment. The effectiveness of this step depends on several factors, including temperature and time. For dried or low-moisture products, heat treatment is less effective. Many tissue products cannot tolerate the temperatures required for viral inactivation, but for those that can, this treatment may be effective for some or all viruses.

- Oxidizing agents. These chemicals can be effective for most viruses, but the effectiveness will vary with the particular agents used.

- Gamma or electron beam irradiation. These steps may be effective for some or all viruses. The dose of irradiation is carefully balanced to prevent damage to the tissue while inactivating the virus effectively. These studies can be complicated to perform, since the material must be shipped to a qualified/permitted vendor for irradiation and then returned to the testing laboratory for virus titration.

- Additional considerations. When performing inactivation steps with biotherapeutics in solution, multiple timepoints are tested to evaluate the kinetics of inactivation. This is preferred for tissue-derived products as well. However, there are some situations where it is not practical to define or to test multiple timepoints. One example is oven-drying, where the process cannot be interrupted.

\section{Scale-Down}

For some products, the first tissue processing step is to grind or mill the material prior to treating with various inactivants. In these cases, it is simple to scale-down the testing process by using small amounts of ground or milled material and inactivant appropriately. For sheets of tissue (e.g., skin or epithelial), small pieces can be trimmed from the larger sheet. However, products like heart valves cannot be cut into smaller 
pieces. An entire valve may be required for each virus/timepoint used, and this will impact the amount of material needed to perform the study.

Tissue-derived products from organs (e.g., pancreas, liver, heart) are comprised of multiple tissue types. Skin and epithelium are non-symmetrical, with different compositions on the two sides. While grinding and milling can improve the homogeneity of tissue products, some micro-heterogeneity will remain and must be considered in the design of the viral clearance study.

Unlike studies with solution-based biotherapeutic products that track multiple timepoints, tissue studies require an independent piece of tissue for each timepoint. The potential for variability between pieces adds to the inherent variability of the results.

When scaling down a processing step, it is important for the small-scale process to be as representative of the fullscale process as possible. With tissue studies, this can be done by using a small amount of tissue and scaling the solution volumes proportionally. However, one parameter that does not scale proportionally is mixing. The same mixing operation in a smaller volume results is a much stronger force on the material, and therefore may be non-representative of the full-scale process. Typically, viral clearance steps are performed in small tubes, and the best form of mixing is rocking or rotating. The mixing speed is chosen to give a level of mixing that appears to be as strong as the full-scale process. However, this is determined by eye and is not an exact determination.

It is not uncommon for issues such as sample handling and virus recovery to impact the study's performance. Therefore, we consider it essential to perform feasibility studies in advance of the clearance study. Any issues that are identified can be addressed by changes in the study design, thus allowing for a smooth performance of the clearance study.

\section{Viruses}

The viruses selected for a viral clearance study should model the kinds of viruses that could be found in the starting material. A thorough risk analysis should be performed to identify these viruses. For materials sourced from human tissue, bloodborne viruses usually represent the highest risks, so the viruses chosen should model them. For materials sourced from bovine or porcine materials, the risks are associated with country of origin, open vs. closed herds, vaccination and animal husbandry practices, and slaughterhouse procedures. Model viruses frequently chosen for viral clearance studies are shown in Table 3. Certain viruses commonly used in viral clearance studies (e.g., bovine viral diarrhea virus) are commonly found in animal herds. If such viruses are present in the tissue, they can complicate the interpretation of the results because the naturally present virus may not behave identically to the lab strain used for spiking. In addition, antibodies to the virus could be present in the tissue, which could interfere with the assay used to quantitate the virus.

\section{Virus Spiking}

The simplest study approach would be to spike virus into the solution containing the same chemicals that were used to process the tissue. However, it is important to perform the studies in a way that is as representative as possible of the actual tissue processing protocols. We recommend spiking a small volume of virus onto a piece of the tissue and incubating it until the virus is adsorbed and the added liquid has evaporated. The spiked tissue is then subjected to the appropriate processing step (e.g., Table 2). Following this, the tissue is treated with an inactivant to neutralize the spiked virus and then washed to remove the inactivant.

For products that are ground or milled, a small sample of tissue can be spiked with virus. For sheets of tissue, a small piece can be cut out and spiked with virus. If the product is a larger object, such as a valve or an organ, it is not possible to spike the tissue evenly. It may be necessary to spike the tissue in several spots in order to represent the different non-homogeneous components in the tissue.

Further considerations related to the complex nature of tissues and viral clearance studies include:

- Prior to spiking, it is important to be sure that the tissue does not contain any components (e.g., solvents, detergents) that could cause viral inactivation. If an inactivant is present, the tissue should be rinsed beforehand. Otherwise, it will be impossible to recover the virus and test the processing step for effectiveness.

- If tissue such as skin is in sheet form, the two non-identical sides of the tissue should be identified before testing. Typically, one side of the tissue will be chosen for spiking if it is more likely to be exposed to virus in the mammal. If both sides are considered vulnerable to virus, then both sides should be tested. It is likely that each side of the tissue will produce different results.

- If the tissue is dry before spiking, the spiking liquid must be entirely gone so that the tissue achieves the same degree

\begin{tabular}{|c|l|}
\hline \multicolumn{2}{|c|}{ TABLE 3. Virus panels used in viral clearance studies. } \\
\hline Tissue Source & \multicolumn{1}{|c|}{ Virus Panel (family) } \\
\hline \multirow{2}{*}{ Human } & Human immunodeficiency virus (lentivirus) \\
\cline { 2 - 3 } & Porcine parvovirus (parvovirus) \\
\cline { 2 - 2 } & Pseudorabies virus (herpesvirus) \\
\cline { 2 - 2 } & Reovirus (reovirus) \\
\cline { 2 - 2 } & Encephalomyocarditis virus (picornavirus) \\
\hline \multirow{2}{*}{ Porcine } & Porcine parvovirus (parvovirus) \\
\cline { 2 - 2 } & Pseudorabies virus (herpesvirus) \\
\cline { 2 - 3 } & Reovirus (reovirus) \\
\cline { 2 - 3 } & Xenotropic murine leukemia virus (retrovirus) \\
\hline \multirow{2}{*}{ Bovine } & Porcine parvovirus (parvovirus) \\
\cline { 2 - 3 } & Pseudorabies virus (herpesvirus) \\
\cline { 2 - 3 } & Bovine viral diarrhea virus (flavivirus) \\
\cline { 2 - 3 } & Xenotropic murine leukemia virus (retrovirus) \\
\hline
\end{tabular}


of dryness as before. This can create challenges for enveloped viruses sensitive to drying.

As noted above, performing the testing in the presence of tissue is preferred, as it is the "worst-case" scenario. However, there are times when this is not possible and the study must be conducted in solution without any tissue. If solution testing must be used, it is best to generate supporting data demonstrating why a study cannot be performed in the presence of tissue.

\section{Virus Recovery}

Once the spiking step is complete, the virus must be recovered from the tissue. In our laboratory, we incubate the tissue in solution with periodic vortexing. This step is usually very effective, but preliminary studies must be conducted in advance to ensure that the incubation is shown to be effective.

Since the processing steps are designed to remove unwanted components, the nature of the tissue is changed at each step. As a result, the ease of virus recovery may be different for each step. There are several reasons why virus may not be recoverable from the tissue:

- If the tissue contains residual inactivant from the previous processing step, the virus may be inactivated immediately upon spiking.

- The virus might not be released during the recovery process because it has bound to the tissue. It may be helpful to adjust the composition of the recovery solution with salt or other chemistries.

- Animals may be vaccinated to prevent common viruses from infecting herds. Tissue from vaccinated or naturally infected animals may contain enough antibody to neutralize the virus. In this case, a similar model virus should be used that is not found naturally in the animal species.

\section{Controls}

It is important to maintain personnel safety protocols when performing the process steps in the presence of virus. For some products, the treatments are performed in an open vessel with mechanical mixing. There are two reasons why this is not acceptable. If virus is present: (1) the mixing can generate aerosols that spread through the air; and (2) an open vessel is a risk for spills. Mixing should be performed in a closed container with a stir bar or other mechanical movement device.

Processes using solvents for viral extraction can create issues with chemical as well as biological safety, and should be performed in a fume hood or class IIB biosafety cabinet. Because of these hazards, an appropriate system should be in place to handle the waste material disposal.

Processes such as high-speed tissue grinding can produce aerosols. Therefore, the process must be performed in a sufficiently sized biosafety cabinet to contain the airborne particulates. Even if the processing equipment is closed during operation, it must be opened in a biosafety cabinet. Otherwise, it may not be possible to evaluate the step for viral clearance.

\section{Conclusions}

Performing viral clearance studies with tissue-derived products can be technically demanding. The steps required to process non-homogeneous tissue materials are often quite different than those used with recombinant DNA products in solution. As such, additional time is required for designing a study, making adjustments, and performing feasibility experiments to verify the performance of the steps chosen. If compromises must be made in a study's design, we recommend that the tissue manufacturer review it with the regulatory authorities in advance to ensure the acceptability of the study.

In a number of products, we have seen that sufficient viral clearance could not be determined because the inactivation steps were ineffective or were not part of the process design. This can be avoided if the purification process is designed with viral clearance in mind. It is recommended that at least two of the treatments listed in Table $\mathbf{2}$ be included in any process design. Unlike biotherapeutics, there are no "modular" steps that can be counted on to provide good clearance in all tissue-derived products because of their distinctly different characteristics.

Despite the challenges involved in performing viral clearance studies with tissue-derived products, satisfactory results can be obtained by planning in advance for well-designed studies and proper execution.

\author{
About the Authors \\ Stephen Stoltzfus, MS*, Manager, Viral Clearance Services \\ Katherine F. Bergmann, PhD, former Manager, \\ Viral Safety and Clearance Services \\ Eurofins Lancaster Laboratories, Inc. \\ 2425 New Holland Pike \\ Lancaster, Pennsylvania 17601 USA \\ *Corresponding Author: \\ Email: stephenstoltzfus@eurofinsus.com \\ Phone: +1 717-556-3104
}

